\title{
Inertial eigenvalues, rod density, and rod diameter in length perception by dynamic touch
}

\author{
CLAUDIA CARELLO, PAULA FITZPATRICK, ITTAI FLASCHER, and M. T. TURVEY \\ University of Connecticut, Storrs, Connecticut
}

\begin{abstract}
Four experiments addressed the relevance of the eigenvalues $I_{k}$ of the inertia tensor for perceiving length by dynamic touch. Experiments 1-2 focused on the consequences of limiting variation in the minimum eigenvalue $I_{3}$. Both revealed that perceived length is a function of $I_{k}$. Whether the contribution of $I_{3}$ is detected, however, depends on the range of values that characterize a particular object set. Experiments 3-4 considered the relationship between an independent index of a rod's diameter, which does not affect $I_{k}$, and actual manipulation of a rod's diameter, which does affect $I_{k}$. Whereas the former appeared as satisfaction of implicit instructions to alter reports of perceived length, the latter entailed actual differences in perceived length in accordance with $I_{k}$. Results are discussed with respect to the links among actual length, perceived length, and $I_{k}$, as well as, in particular, how these links guarantee that perceived length is in the range of actual lengths.
\end{abstract}

When an object is grasped firmly in the hand and wielded out of view by means of movements at the wrist, ts length can be perceived. A large number of experiments lave shown that perceived length is a function not of the sbject's actual length but rather of its resistance to rotaional acceleration as quantified by the inertia tensor, $I_{i j}^{l}$ see Appendix). In the initial research directed at the spaial abilities of dynamic touch, measurements were reitricted to the coordinate-system-dependent components of $I_{i j}$, and then only to its moments of inertia, $I_{x x}, I_{y y}$, and ${ }_{z z}$ (e.g., Solomon \& Turvey, 1988; Solomon, Turvey, \& Zurton, 1989a, 1989b). As the research progressed, the ignificance of the products of inertia-the off-diagonal erms in the $3 \times 3$ matrix representation of $I_{i j}$-became ıpparent (e.g., Pagano \& Turvey, 1992; Turvey, Burton, 'agano, Solomon, \& Runeson, 1992), together with the leed to compute the coordinate-system-independent orm of $I_{i j}$. This computation is done by diagonalizing $I_{i j}$ Borisenko \& Tarapov, 1979; Goldstein, 1980; Kibble, 1985), essentially referring the rotational inertia to the rincipal axes or eigenvectors $\mathbf{e}_{k}(k=1,2,3)$ about which he off-diagonal terms disappear. The diagonal terms are he principal moments or eigenvalues $I_{k}$, representing the naximal moment of inertia $I_{1}$, the minimal moment of nertia $I_{3}$, and an intermediate moment of inertia $I_{2}$ (for iylindrical symmetry, $I_{2}=I_{1}$, otherwise $I_{2} \neq I_{1}$ ). The ame $\mathbf{e}_{k}$ and $I_{k}$ result from diagonalization regardless of he coordinate system chosen at the fixed point of wieldng and, therefore, the particular moments and products omposing $I_{i j}$.

The research reported here was supported by Grant SBR 93-09371 rom the National Science Foundation. C.C. and M.T.T. are also at Hasins Laboratories, New Haven, CT. Correspondence should be addressed o C. Carello, CESPA, U-20, 406 Babbidge Road, University of Connecti:ut, Storrs, CT 06269-1020 (e-mail: cespa1@uconnvm.uconn.edu).
Although a number of results on haptically perceiving the length of objects with nearly cylindrical symmetry are readily accommodated by the major eigenvalue $I_{1}$ alone (see, e.g., Pagano, Fitzpatrick, \& Turvey, 1993; Pagano \& Turvey, 1993), for the more general case (i.e., objects of any symmetry), perception of linear extents must be a function of $I_{k}$ (Fitzpatrick, Carello, \& Turvey, 1994). This is because the equation for rotations about any arbitrarily chosen axis is

$$
I_{1} \dot{\omega}_{1}-\omega_{2} \omega_{3}\left(I_{2}-I_{3}\right)=N_{1},
$$

together with two similar equations obtained by cyclic permutation of 1,2 , and 3 (where $N$ is torque, $\omega$ is angular velocity, and the overdot stands for the first timederivative).

Fitzpatrick et al.'s (1994) hypothesis was thus that, in the general case, the perception of the linear dimensions of an object by wielding will be specific to the inertia tensor. They reported four experiments that provided strong confirmation of this hypothesis. Experiments 1-2 manipulated rod diameter and density, respectively. Experiment 3 decoupled $I_{1}$ and $I_{3}$ (through judicious combinations of rod diameter, handle diameter, and attached masses), and Experiment 4 manipulated $I_{1}$ and $I_{3}$ without any change in the diameter at the point of grasp by attaching a handle of fixed dimensions to the base of each of five types of geometric objects (hemisphere, cylinder, parallepiped, pyramid, and cone) of three different sizes (for a total of $15 \mathrm{ob}-$ jects). In each experiment, perceived length was found to be a reliable function of $I_{1}$ (raised to a positive exponent) and $I_{3}$ (raised to a negative exponent) $\left(r^{2}=.99, .99, .99\right.$, and .93 for Experiments 1-4, respectively).

In a recent article on the nonvisible perception of an object's length by dynamic touch, Chan (1995) disputed this conclusion: "There is no convincing evidence that $I_{3}$ is a covariant of $I_{1}$ for perception of rod length" (p. 784). In the place of $I_{3}$ he proposed to substitute diameter 
per se, provided by muscular tension accompanying grasps of different sizes and independent knowledge about that diameter, provided either by the size of the grasp or by a reference segment grasped in the other hand. $\mathrm{He}$ termed these "muscular" and "geometric" constraints, respectively, and considered them as modifiers of the perception of length that is tied to the physical invariant $I_{1}$. The experiments of Fitzpatrick et al. (1994), however, had already ruled out diameter. Whether the latter was varied or held constant, perceived length was determined by $I_{1}$ and $I_{3}$. When one considers the four experiments together, Chan's hypothesis that length perception is a function of $I_{1}$ and diameter accommodates only $28 \%$ of the variance in the 48 mean perceived lengths, whereas Fitzpatrick et al's hypothesis that length perception is a function of $I_{1}$ and $I_{3}$ accommodates $97 \%$ of the variance. Figure 1a reproduces Figure 7 from Fitzpatrick et al., which demonstrates this ability of the major and minor eigenvalues to predict perceived length across the 48 very different objects of their four experiments. Figure $1 \mathrm{~b}$ shows the contrasting poor support for the diameter hypothesis for all objects. ${ }^{2}$

Chan (1995) was led to his hypothesis because his Experiment 1 failed to find a significant contribution of $I_{3}$ with rods that differed in density. As noted, Fitzpatrick et al. (1994) had found such a contribution in an experiment in which rods of different densities (wood, aluminum, and steel) were inserted in identical wooden handles of a fixed diameter. Chan's experiment used very thin rods (less than $1 \mathrm{~cm}$ in diameter) of only two densities (aluminum and steel). It is likely that these factors minimized the potential contribution of $I_{3}$. Each eigenvalue is affected by how far the mass is from the point of rotation along the relevant axis. For $I_{3}$, this distance is the rod radius. If the radius is small and the mass variation is limited, the variation in $I_{3}$ will be limited. For $I_{1}$, those same mass differences are multiplied by much larger distances - up to half the length of the rod. For certain object sets, therefore, the contribution of $I_{1}$ may overwhelm the contribution of $I_{3}$ in a multiple regression. Because Chan's rods were homogeneous throughout their length (i.e., there were no handles), the density experiment of Fitzpatrick et al. has been replicated here without handles. We expected to verify that the range of eigenvalues, not assumptions about material or diameter that participants may have derived from the thick wooden handles, determines whether the contribution of $I_{3}$ is apparent.

The present Experiment 2 ran the preceding argument in reverse. Can we take a set of objects that have shown a contribution of $I_{3}$ and eliminate it by somehow restricting the variation in $I_{3}$ ? Fitzpatrick et al. (1994) showed that for rods of different diameter, the variation in $I_{3}$ is substantial and its contribution is significant. If those same rods are inserted into handles of fixed outer diameter, the consequence for $I_{3}$ is to restrict the variation brought about by the diameter of the rods themselves. The variation in $I_{1}$, in contrast, remains substantial. Experiment 2 examined whether the same rods that produced a significant contribution of $I_{3}$ in Fitzpatrick a
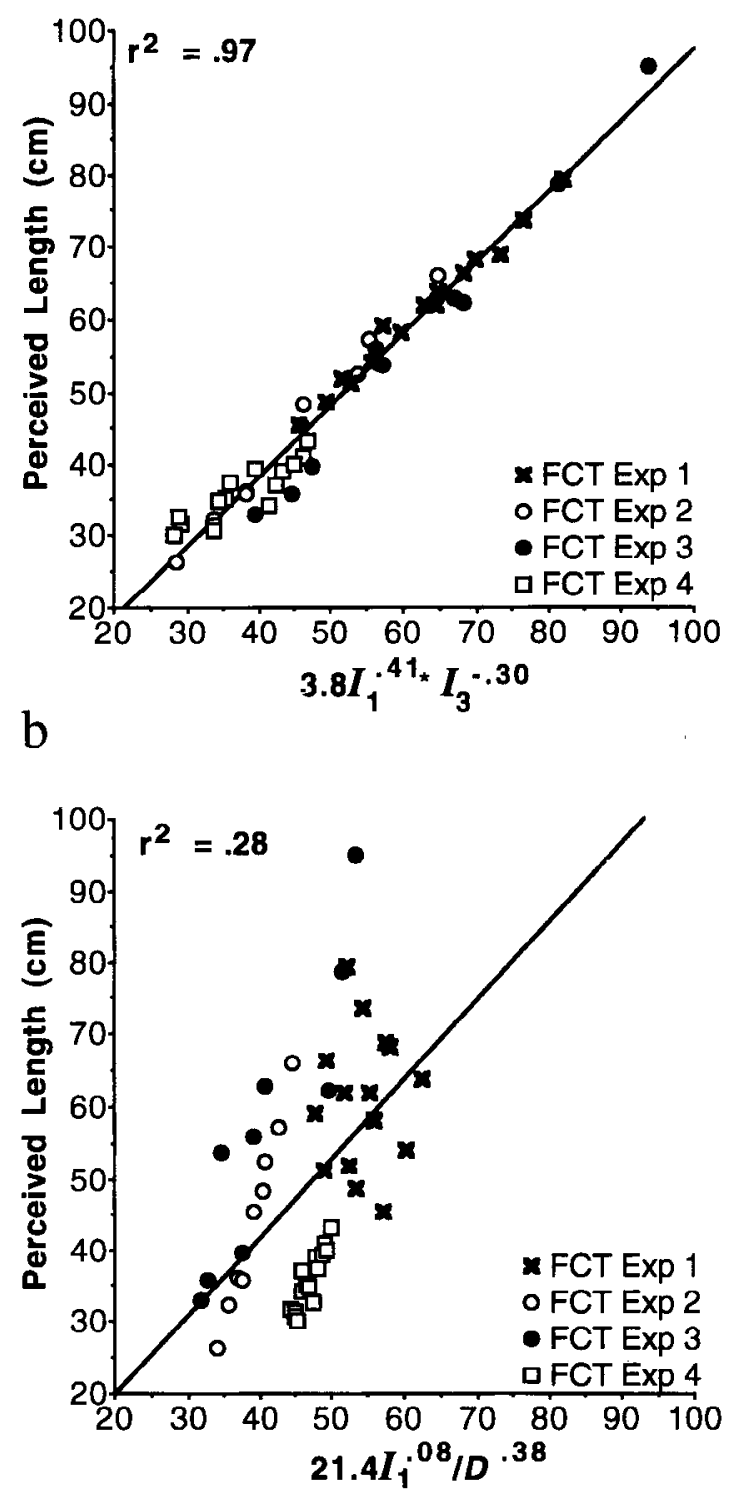

Figure 1. Perceived length for the data of the four experiments in Fitzpatrick et al. (1994) as a function of two regression models. (a) The model $3.8 I_{i}^{41} I_{3}^{-30}$, derived from the multiple regression of $\log$ perceived length on $\log I_{1}$ and $\log I_{3}$, was suggested by Fitzpatrick et al. from tensorial considerations. (b) The model 21.4 $I_{i}{ }^{(08} d^{-.38}$, derived from the multiple regression of log perceived length on $\log I_{1}$ and $\log$ diameter, was suggested by Chan (1995) from a consideration of the muscular and geometric consequences of grasping a rod.

et al. would fail to do so under the restricted variation in $I_{3}$ brought about by the addition of handles.

Experiments 3 and 4 addressed Chan's (1995) conjecture about the contribution of diameter. He noted that diameter has both muscular and geometric consequences for the grasping hand. That is, increased diameter is accompanied by greater tissue deformation (through the 
increase in mass) as well as "knowledge about diameter" (through the size of the grasp). He argued that when the knowledge is obtained by means of the diameter of a reference segment grasped by the nonwielding hand, it affects perceived length in the same way (his Experiment 3 ). The present Experiment 3 examined the metrical consequences of such reference segments and compared them with the metrical consequences of real diameter manipulations in Experiment 4.

\section{EXPERIMENT 1}

In Chan's (1995) Experiment 1, the largest $I_{3}$ was three times the size of the smallest. ${ }^{3}$ In the density experiment of Fitzpatrick et al. (1994), these differed by a factor of 6. Limiting the analysis of those data to the aluminum and steel rods reduces the $I_{3}$ variation to Chan's range (a factor of 3.2) and eliminates the significant contribution of $I_{3}(p>.10)$. In Fitzpatrick et al.'s experiment, every rod was inserted into the same wooden handle of $2.4-\mathrm{cm}$ diameter. Given Chan's argument that knowledge about the rods is obtained through the grasping hand, one might conjecture that participants thought that all of the rods were wood and twice their actual diameter. If they modulated their responses accordingly, this might have appeared as a spurious contribution of $I_{3}$. The density experiment of Fitzpatrick et al. has been replicated here, therefore, without handles. Participants could feel the differences among the compositions (e.g., through surface temperature) and, presumably, could use that knowledge to mitigate the contribution of $I_{3}$.

\section{Method}

Participants. Six undergraduates at the University of Connecticut volunteered to participate in partial fulfillment of a course requirement.

Materials. Solid cylinders of three materials (wood, aluminum, and steel), three lengths $(37,46$, and $55 \mathrm{~cm})$, and fixed diameter $(1.2 \mathrm{~cm})$ were used. Average masses for the three types were 39.1 , 156.8 , and $453.3 \mathrm{~g}$. The tensors were calculated relative to a rotation point in the wrist that was taken to be $6 \mathrm{~cm}$ from the longitudinal $(z-)$ axis of the rod. They were diagonalized using the Jacobi transformation method (Sprott, 1991). The maximum and minimum eigenvalues for each rod are shown in Table 1.

Apparatus. The participant sat at a student desk with a surface to support the right arm. Wielded rods were occluded by an opaque curtain to the participant's right. A motorized surface in front of the participant could be positioned anywhere from 0 to $2.3 \mathrm{~m}$ by means of foot pedals (Figure 2).

Procedure and Design. Three repetitions of nine combinations (three rods and three densities) were presented in random order. No information was provided about the lengths, shapes, number of objects, or where they were grasped, and no practice or feedback was given. The experimenter placed a rod in the participant's right hand, with the instruction to grasp it firmly and limit movements to the wrist. The participant was allowed to wield the rod for as long as needed. Perceived length was indicated by moving the visible surface to a position that coincided with this perceived length.

\section{Results and Discussion}

Mean perceived length is shown in the far-right column of Table 1. A 3 (length) $\times 3$ (density) within-subjects
Table 1

Object Density, Length, $I_{1}, I_{3}$, and

\begin{tabular}{|c|c|c|c|c|}
\hline $\begin{array}{l}\text { Object } \\
\text { Density }\end{array}$ & $\begin{array}{l}\text { Length } \\
(\mathrm{cm})\end{array}$ & $\begin{array}{c}I_{1} \\
\left(\mathrm{~g} \cdot \mathrm{cm}^{2}\right) \\
\end{array}$ & $\begin{array}{c}I_{3} \\
\left(\mathrm{~g} \cdot \mathrm{cm}^{2}\right)\end{array}$ & $\begin{array}{c}\text { Perceived } \\
\text { Length }(\mathrm{cm})\end{array}$ \\
\hline Wood & $\begin{array}{l}37 \\
46 \\
55\end{array}$ & $\begin{array}{l}17,748 \\
28,155 \\
48,785\end{array}$ & $\begin{array}{l}299 \\
330 \\
412\end{array}$ & $\begin{array}{l}23.0 \\
28.0 \\
32.9\end{array}$ \\
\hline Aluminum & $\begin{array}{l}37 \\
46 \\
55\end{array}$ & $\begin{array}{r}62,047 \\
116,257 \\
195,936\end{array}$ & $\begin{array}{l}1,094 \\
1,390 \\
1,681\end{array}$ & $\begin{array}{l}25.0 \\
31.7 \\
36.7\end{array}$ \\
\hline Steel & $\begin{array}{l}37 \\
46 \\
55\end{array}$ & $\begin{array}{l}179,689 \\
336,018 \\
565,874\end{array}$ & $\begin{array}{l}3,169 \\
4,016 \\
4,856\end{array}$ & $\begin{array}{l}32.6 \\
40.5 \\
48.3\end{array}$ \\
\hline
\end{tabular}

analysis of variance (ANOVA) revealed an effect of rod density $[F(2,10)=22.68, p=.0002$; wood, aluminum, and steel averaged $27.99,31.11$, and $40.49 \mathrm{~cm}$, respectively] and rod length $[F(2,10)=39.88, p=.0001 ; 37-$, $46-$, and $55-\mathrm{cm}$ rods averaged $26.88,33.42$, and $39.30 \mathrm{~cm}$, respectively], but not their interaction $[F(4,20)=2.11$, $p>.10]$.

The multiple regression of log perceived length on $\log I_{1}$ and $\log I_{3}$ revealed both components to be significant $\left(r^{2}=.95, p<.001\right)$, with a positive exponent on $I_{1}$ (.45, Partial $F=46.14)$ and a negative exponent on $I_{3}$ $(-.30$, Partial $F=17.72)$. These results support the claim that the effectiveness of $I_{3}$ in Experiment 2 of Fitzpatrick et al. (1994) was due to the range of eigenvalues and not to misconceptions about their material or diameter. To be precise, $I_{1}$ and $I_{3}$ underlie perception of length in all instances, but showing this statistically requires substantial variation in $I_{3}$ relative to $I_{1}$.

\section{EXPERIMENT 2}

If variation in the range of $I_{3}$ is needed to reveal its contribution, then that contribution should be eliminated when the variation is restricted somehow. Fitzpatrick et al. (1994) showed that using rods of three lengths and five diameters produces adequate variation: The largest $I_{3}$ was 13 times greater than the smallest in their Experiment 1 . If those same rods are inserted in handles of a fixed diameter and of the same material, then the largest $I_{3}$ is only 1.7 times greater than the smallest. The variation in $I_{1}$ is also reduced by this addition, but it is still substantial (from a factor of 27 to a factor of 15). With such objects, we would expect perceived length to be a function of $I_{1}$ only.

\section{Method}

Participants. Eight undergraduates at the University of Connecticut volunteered to participate in partial fulfillment of a course requirement.

Materials. Wooden rods of five diameters $(.50, .70, .80,1.00$, and $1.28 \mathrm{~cm})$ and three lengths $(61,76$, and $91 \mathrm{~cm})$ were inserted into $2.30-\mathrm{cm}$-diameter wooden handles $15 \mathrm{~cm}$ long. The handle was hollowed just enough to accommodate the diameter of the rod. The average masses of the rods were $7.73,15.30,24.23,32.50$, and $61.97 \mathrm{~g}$ for the five diameters. The corresponding handle masses were 54.8 , 


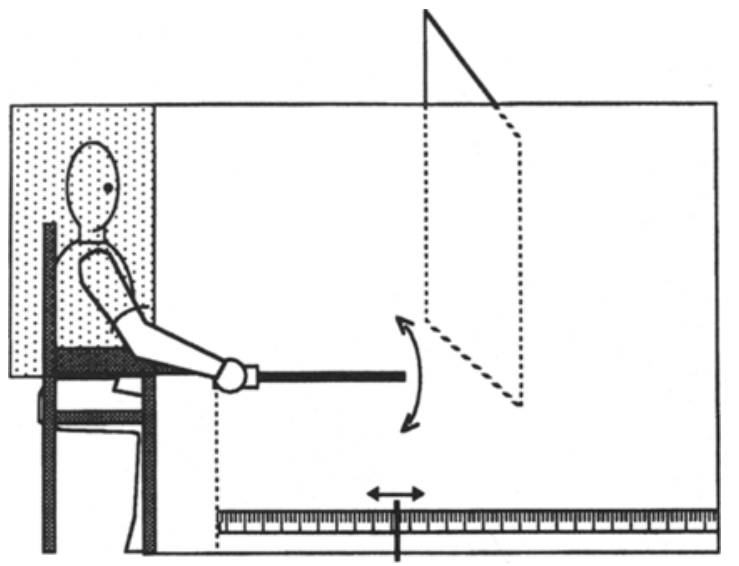

Figure 2. The participant wields a rod occluded from view. A marker visible to the experimenter indicates the distance of a surface that has been positioned to report perceived length.

$51.0,43.3,47.2$, and $38.5 \mathrm{~g}$ (i.e., handle mass decreased as more was hollowed out to accommodate wider rods). Using the parallel axis theorem (see, e.g., Goldstein, 1980), the tensors were calculated separately for the rods and handles, added together, and diagonalized to yield the eigenvalues. The maximum and minimum eigenvalues for each rod are shown in Table 2.

Apparatus, Pmcedure, and Design. The apparatus, procedure, and design were the same as in Experiment 1 except that there were 15 combinations (three lengths and five rod diameters).

\section{Results and Discussion}

Mean perceived lengths are shown in the far-right column of Table 2. A 5 (diameter) $\times 3$ (length) ANOVA revealed an effect of rod diameter $[F(4,28)=29.75, p<$ $.0001]$ and rod length $[F(2,14)=47.34, p<.0001]$, as well as their interaction $[F(8,56)=4.73, p<.0002]$. Perceived length increased with increases in rod length and with increases in rod diameter. The effect of diameter was accelerated for the longer rods but was apparent for all.

The multiple regression of log perceived length on $\log I_{1}$ and $\log I_{3}$ revealed that the contribution of $I_{3}$ was not significant (Partial $F=2.03, p>.15$ ). The simple regression on $I_{1}$ was sufficient $\left(r^{2}=.96, p<.0001\right)$. The effect of diameter was similar to its effect in Experiment 1 of Fitzpatrick et al. (1994) in that perceived length increased with increases in diameter. As the equations in the Appendix show, increasing the diameter increases the diagonal components directly (and increases all of the tensor components indirectly through the increase in mass). As expected, however, reducing the range of $I_{3}$ values rendered the overall contribution of $I_{3}$ unreliable, so this effect is largely anchored in $I_{1},{ }^{4}$

The present Experiments 1 and 2 buttress the conclusion of Fitzpatrick et al. (1994) that perceived length by dynamic touch is a function of $I_{k}$. We have established further that whether perceived length reveals a contribution of the minimum eigenvalue depends on the extent of variation in that component. For the objects of Experiment 2 , the values generated by the power equation using $I_{1}$ alone are predicted tightly by the values generated using the power equation from Fitzpatrick et al., which requires both eigenvalues $\left(r^{2}=.99\right)$. A similar comparison for those same objects without handles (Experiment 1 of Fitzpatrick et al.) reveals a difference between the single and double eigenvalue power equations $\left(r^{2}=\right.$ .77). If $I_{3}$ does not vary, it cannot modulate the values generated by the double eigenvalue power equation- $I_{1}^{\mathrm{a}}$ is, in effect, being multiplied by a constant - and it cannot be significant. To illustrate, the exponent for $I_{1}$ was similar in the two experiments - .45 and .47 , respectively. In Experiment 1 (as well as in Fitzpatrick et al., 1994), the exponent on $I_{3}$ was -.30 . For Experiment $1, I_{3}^{-.30}$ produced values ranging from .08 to .18; for Experiment 2, $I_{3}^{-.30}$ produced values ranging only from .11 to .13 . The contribution of $I_{3}$ was not detected in the latter case, but $I_{k}$ is nonetheless the constraint on perceived length.

Chan (1995) identified an additional constraint on perceived length that he called "knowledge about its diameter." In his Experiment 3, participants were misled about the diameter of the wielded rod by means of a segment grasped in the nonwielding hand. Chan found that for a rod of a given diameter wielded in the right hand, perception of its length was inversely related to the diameter of a segment grasped in the left hand. This contribution was independent of the influence of the wielded rod's actual diameter, which, for rods of fixed $I_{1}$, was also inversely related to perception of its length.

Chan (1995) described these influences as the integration of different constraints into a single perception. Most significantly, he considered the contribution of segment diameter as a demonstration of the influence of knowledge on perception. This interpretation is premature, however. Although it is the case that reports of length were influenced by the diameter of the reference segment, this is precisely what the instructions requested. Participants may have simply perceived length by wielding and then modulated their responses categorically for the three segment diameters (notwithstanding the experimenter's admonition to "perceive directly and intuitively"). To conclude that knowledge gleaned from the segment constrained perception of the wielded rod re-

Table 2

Object Diameter, Length, $I_{1}, I_{3}$, and

Mean Perceived Length in Experiment 2

\begin{tabular}{ccccc}
\hline $\begin{array}{c}\text { Diameter } \\
(\mathrm{cm})\end{array}$ & $\begin{array}{c}\text { Length } \\
(\mathrm{cm})\end{array}$ & $\begin{array}{c}I_{1} \\
\left(\mathrm{~g} \cdot \mathrm{cm}^{2}\right)\end{array}$ & $\begin{array}{c}I_{3} \\
\left(\mathrm{~g} \cdot \mathrm{cm}^{2}\right)\end{array}$ & $\begin{array}{c}\text { Perceived } \\
\text { Length }(\mathrm{cm})\end{array}$ \\
\hline .50 & 61 & 13,994 & 1,031 & 24.64 \\
& 76 & 22,119 & 1,287 & 27.11 \\
& 91 & 30,788 & 1,466 & 39.17 \\
70 & 61 & 22,345 & 1,209 & 25.96 \\
& 76 & 36,337 & 1,431 & 34.49 \\
& 91 & 54,170 & 1,415 & 45.65 \\
80 & 61 & 29,694 & 1,112 & 30.11 \\
& 76 & 54,456 & 1,329 & 41.58 \\
& 91 & 82,684 & 1,464 & 57.69 \\
1.00 & 61 & 36,027 & 1,188 & 32.74 \\
& 76 & 68,865 & 1,555 & 50.55 \\
& 91 & 119,326 & 1,732 & 61.02 \\
1.30 & 61 & 74,202 & 1,400 & 49.90 \\
& 76 & 128,132 & 1,600 & 67.65 \\
& 91 & 218,170 & 1,763 & 78.52 \\
\hline
\end{tabular}


quires minimally that perceived length be constrained not just categorically but proportionally by the size of the segment, at least insofar as that knowledge is intended to account for data patterns implicating $I_{3}$. Perceived length should differ for two different sets of reference diameters, one thin relative to the other. This prediction of the knowledge hypothesis was tested in the present Experiment 3. A second implication of the knowledge hypothesis is that the source of knowledge about diameter should be irrelevant in constraining perception; whether it comes from reference segments or from wielded rods, perceived length should be affected in the same way. To provide such a comparison for the segment-derived knowledge of Experiment 3, Experiment 4 examined perceived length of two sets of rods whose diameters actually differed, with one set thin relative to the other.

\section{EXPERIMENT 3}

A variant of Chan's (1995) Experiment 3 was conducted with two groups of participants. The actual rods to be perceived were the same for the two groups: $60-\mathrm{cm}$ rods of $.50-, .90-$, and $1.20-\mathrm{cm}$ diameter inserted in a handle $2.30 \mathrm{~cm}$ in diameter. Group 1 was provided with three reference segment diameters: $.50, .90$, and $1.20 \mathrm{~cm}$. Group 2 was provided with three relatively thicker reference segment diameters: $1.20,1.80$, and $2.30 \mathrm{~cm}$. Within each group, perceived length was expected to increase with actual diameter, as in Experiment 2 and Fitzpatrick et al. (1994), but to decrease with reference segment diameter, as in Chan. Across groups, if perceivers "use knowledge" about segment diameter, then Group 2 should produce smaller perceived lengths than Group 1. The segments are larger, so their consequences should be exaggerated. In addition, the thickest segment of Group 1 should be associated with the same perceived rod length as the thinnest segment of Group 2, given that they involve the same combination of rod diameter and reference segment diameter. Alternatively, if perceivers simply follow instructions to modulate perceived length by virtue of what is in the left hand, without using the realsize consequences of the reference segment diameter, then the two groups should not differ overall given that the wielded rods do not differ. Moreover, the thickest segment of Group 1 should be associated with smaller perceived rod lengths than the thinnest segment of Group 2-even though they are the same size-because of how they compare with the other reference segments in their group.

It should be noted that this experiment did not use Chan's (1995) instructions. He told participants that they were wielding a rod in a handle when, in fact, they were wielding homogeneous, cylindrical rods. In addition, he told them that the reference segment provided the actual diameter of that rod when, in fact, it indicated diameters that could be wider or narrower than the actual rods. We avoided misleading participants by asking them to "imagine that the wielded rod is the diameter of the segment in the left hand." With this change in instruction, therefore, we must also examine the extent to which our results mimic Chan's. If reference segments have a similar effect in the two experiments, the claim that this effect is not strictly perceptual will be supported.

\section{Method}

Participants. Fourteen undergraduates at the University of Connecticut volunteered to participate in partial fulfillment of a course requirement. They were assigned randomly to one of the two groups.

Materials. Three $60-\mathrm{cm}$-long wooden rods of three diameters $(.50, .90$, and $1.20 \mathrm{~cm})$ were inserted into $2.30-\mathrm{cm}$-diameter handles $15 \mathrm{~cm}$ long. The handle was hollowed just enough to accommodate the diameter of the rod. The rod masses were $6.0,23.6$, and $52.3 \mathrm{~g}$, and the corresponding handle masses were 52.3, 47.2, and 32.0 . Reference segments for Group 1 were $.50, .90$, and $1.20 \mathrm{~cm}$; reference segments for Group 2 were $1.20,1.80$, and $2.30 \mathrm{~cm}$.

Apparatus. The apparatus was the same as in the previous experiments except for the addition of reference segments, which were arranged vertically and supported by a wooden base within view of and to the left of the participant (Figure 3).

Procedure and Design. Three repetitions of nine combinations (three rods and three reference segments) for each group were presented in random order. The experimenter placed a rod in the participant's right hand and indicated which of the reference segments to grasp. The person was told to wield the rod and handle in the right hand and imagine that the rod was the diameter of the segment in the left hand. Perceived length was indicated by moving the surface to a position that coincided with this perceived length. Participants could take as long as they needed; the segment was grasped as long as the rod was wielded. Reference segment group was a between-subjects variable; rod diameter and relative segment diameter were within-subjects variables.

\section{Results and Discussion}

A 2 (group) $\times 3$ (segment) $\times 3($ rod $)$ ANOVA revealed an effect of rod diameter $[F(2,24)=71.77, p<.0001]$ and segment diameter $[F(2,24)=19.16, p<.0001]$, as well as their interaction $[F(4,48)=6.78, p<.0002]$. Table 3 presents the mean perceived lengths as a function of actual diameter and reference segment diameter for each group and indicates an increase in perceived length with an increase in actual diameter, replicating Fitzpatrick et al. (1994), and a decrease in perceived length with an increase in segment diameter, replicating Chan (1995). The segment influence was slightly accelerated

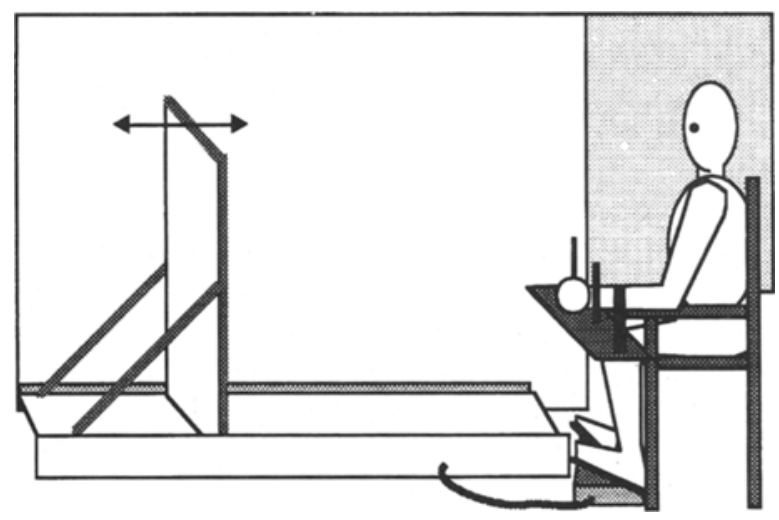

Figure 3. Reference segments were grasped with the participant's left hand in Experiment 3. 
Table 3

Mean Perceived Length as a Function of Actual Rod Diameter, Reference Segment Diameter, and Group in Experiment 3

\begin{tabular}{cccc}
\hline \multirow{2}{*}{$\begin{array}{c}\text { Segment } \\
\text { Diameter }(\mathrm{cm})\end{array}$} & \multicolumn{3}{c}{ Actual Rod Diameter $(\mathrm{cm})^{*}$} \\
\cline { 2 - 4 } & 50 & .90 & 1.20 \\
\hline \multicolumn{5}{c}{ Thinner Set } \\
.90 & 29.1 & 48.1 & 66.4 \\
1.20 & 24.2 & 37.7 & 52.0 \\
& 21.9 & 33.6 & 48.1 \\
1.20 & Thicker Set & & \\
1.80 & 30.5 & 45.9 & 66.8 \\
2.30 & 25.9 & 38.4 & 50.9 \\
\hline
\end{tabular}

*Each rod was inserted in a handle $15 \mathrm{~cm}$ long and $2.30 \mathrm{~cm}$ in diameter.

for the thinnest segment in each group. In contradiction of the knowledge hypothesis, however, the groups did not differ from each other (main effect and all interactions produced $F \mathrm{~S}<1$ ). The two thin, two medium, and two thick reference segments had equivalent influences on reported length, even though the segments for Group 1 were half the diameter of the segments for Group 2. To highlight this equivalence, Figure 4 shows the data averaged across reference segments in each group. Reference segment group did not change perceived length of the rods. It can be seen here and in Table 3 that the thickest segment of Group 1 (1.20-cm diameter) was associated with smaller reports of perceived rod length than was the thinnest segment of Group 2 (1.20-cm diameter), even though they should have provided the same knowledge about diameter.

Clearly, reports of perceived length differed as a function of what a participant felt with the left hand. But if knowledge about the reference segment's diameter was used to modify the perceived length of the wielded rods, the consequences for the two groups should have been different. Reports of perceived length should have continued to decrease for the thicker set of reference segments, with the segment size common to the two groups yielding roughly equivalent values. Instead, the consequences of the two sets of segments were quite independent of each other. Thus, the reference segment manipulation seems to have brought about a response-based rather than a stimulus-based effect (Wedell, 1995). Historically, this kind of response bias has been regarded as an experimental artifact (Anderson, 1975; Stevens, 1971).

\section{EXPERIMENT 4}

It seems likely that participants in Experiment 3 were following implicit instructions to produce, categorically, three different length reports for the same perceived length. If this interpretation is correct, the consequences of altering actual rod diameter should be quite different. Two sets of rods were used, one the same as in Experiment 3 and one relatively thicker. These were presented to two different groups of participants. There were no reference segments. As in Experiment 3, the size of the grasp was always $2.30 \mathrm{~cm}$. The tensor hypothesis asserts that information about length is tied to the mass distribution of the rods themselves. Because the relatively thinner rods of Group 1 were, indeed, smaller than the relatively thicker rods of Group 2, perceived length should be shorter for the former. In addition, the thickest rod of Group 1 should be perceived as the same as the thinnest rod of Group 2, given that they have the same mass distribution.

\section{Method}

Participants. Twelve undergraduates at the University of Connecticut volunteered to participate in partial fulfillment of a course requirement. They were assigned randomly to one of the two groups.

Materials. The rods and handles from Experiment 3 were used as the relatively thinner set. The $1.20-\mathrm{cm}$ diameter rod was also used in the relatively thicker set, along with a $1.80-\mathrm{cm}$-diameter rod, both inserted into the same kind of $2.30-\mathrm{cm}$ diameter handle, and a $2.30-\mathrm{cm}$ rod, which required no handle. The rod masses for Group 2 were $52.3,96.4$, and $183.2 \mathrm{~g}$, and the corresponding handle masses were $32.0,18.0$, and $0 \mathrm{~g}$ (i.e., the $2.3-\mathrm{cm}$ diameter rod had no handle). Tensors for the rods were calculated and diagonalized as in Experiment 2.

Apparatus. All but the reference segments from Experiment 3 were used again.

Procedure and Design. Three repetitions of the three rods for each group were presented in random order. The person was told to wield the rod and adjust the surface to a position that coincided with perceived length. Participants could take as long as they needed. Average rod diameter (thin set vs. thick set) was a between-subjects variable; relative rod diameter (thin, medium, and thick within a set) was a within-subjects variable.

\section{Results and Discussion}

A 2 (group) $\times 3$ (rod) ANOVA revealed an effect of group $[F(1,10)=5.78, p<.05]$ and rod diameter $[F(2,20)=128.96, p<.0001]$, but not their interaction $(F<1)$. Perceived length increased with an increase in

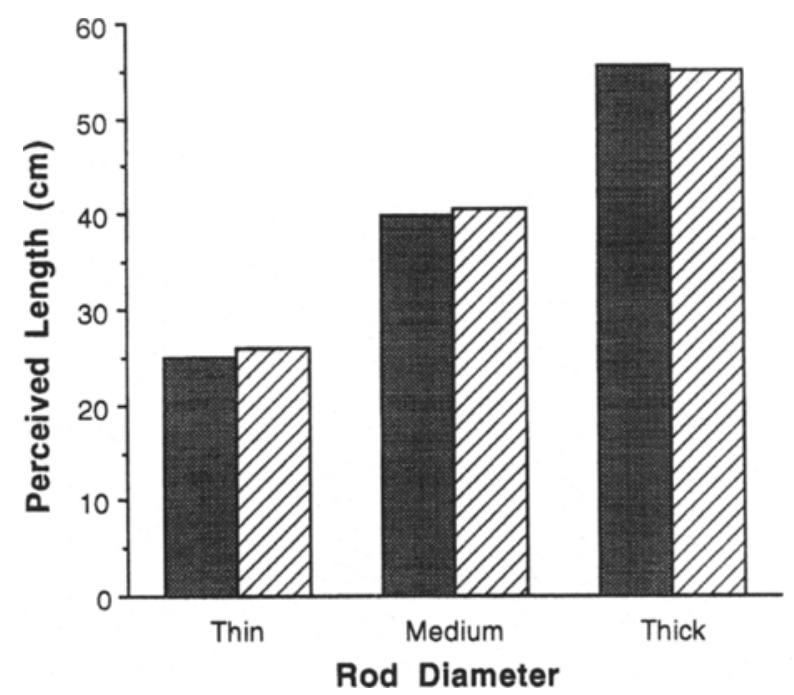

Figure 4. Mean perceived length as a function of actual rod diameter for thin reference segments (dark bars) and thick reference segments (striped bars) in Experiment 3. Reports have been averaged over the three reference segments in each group. 
actual diameter, with the thicker set continuing the increase relative to the thinner set. An examination of the rod common to the two groups indicated that perceived length for the thickest rod for Group 1 and the thinnest rod for Group 2 did not differ $[t(10)=1.5]$. Figure 5 shows the mean perceived lengths for each group. Comparison with Figure 4 shows that altering rod diameter physically was not treated the same way by participants as altering so-called knowledge about rod diameter.

Given that these rods were similar in construction to those in Experiment 2, it was expected that $I_{3}$ would not reach significance. As before, if $I_{3}$ does not vary adequately, it simply stands as a constant multiplier of $I_{1}$ in the power equation and it cannot be significant. A multiple regression of $\log$ perceived length on $\log I_{1}$ and $\log I_{3}$ verified that only $I_{1}$ was significant $\left(r^{2}=.93\right)$, with an exponent of .30 . As will be seen in what follows, these data, together with those from Experiments 1 and 2, are situated appropriately relative to the broad range of objects that have been examined, verifying that $I_{k}$ is the constraint on perceived length.

\section{GENERAL DISCUSSION}

Four experiments addressed the relevance of $I_{k}$ for perceiving length by dynamic touch. The first two experiments focused on the consequences of limiting variation in the minimum eigenvalue. Both found that perceived length is a function of $I_{k}$. Whether $I_{3}$ reaches significance depends on the range of values that characterize a particular experimental set. In particular, if the variation is large (Experiment 1 ), the contribution of $I_{3}$ can be detected; if the variation is limited (Experiment 2), the contribution of $I_{3}$ may not be detected. To reiterate, in the full power equation (i.e., not expressed in log terms),

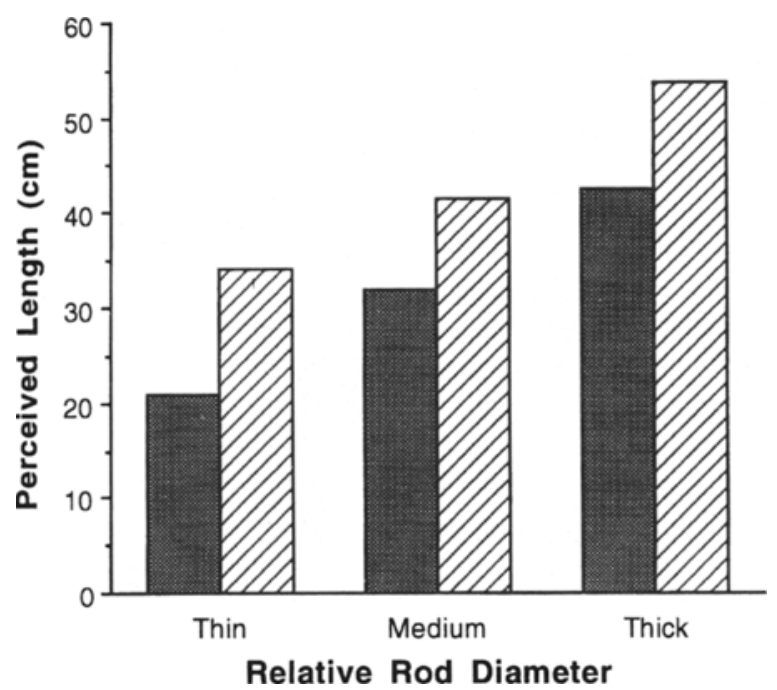

Figure 5. Mean perceived length as a function of actual rod diameter for two groups in Experiment 4 . The smaller rods (dark bars) were $.50, .90$, and $1.20 \mathrm{~cm}$ in diameter; the large rods (striped bars) were $1.20,1.80$, and $2.30 \mathrm{~cm}$ in diameter.
$I_{1}^{a}$ is, in effect, being multiplied by $I_{3}^{-b} \approx$ constant. In Experiments 3 and 4 , we considered the relationship between an independent index of a rod's diameter and the (muscular cutaneous) tissue deformation consequences of a rod's diameter. Although the independent index does indeed alter reports of perceived length, it appears that the consequences of diameter "knowledge" (Experiment 3) are not the same as the consequences of diameter information - that is, information provided through the muscle and skin deformation consequences of the rods' mass distributions (Experiment 4).

Chan (1995) couched his results in terms of constraints applied to different parts of the body being integrated in perception. Whether knowledge about diameter came from grasp diameter of the wielding hand or the nonwielding hand, it was integrated with whatever other information was detected during wielding. As an example of such integration, he noted that a rod's length can be perceived when the rod is supported on the knee with the balancing downward force provided by the edge of one hand (Carello, Fitzpatrick, Domaniewicz, Chan, \& Turvey, 1992). As he correctly pointed out, however, the tissue deformation in those experiments, unlike that registered from his reference segments, was brought about by the rod to be perceived. The conclusion of the Carello et al. (1992) experiments was that the mass distribution, not the particular tissue contact, was important. That is why perceived length with hand and knee was equivalent to using one grasping hand, the top of one hand and the bottom of another, and even the edge of one finger as the only tissue contact (with an environmental support providing the balancing force). These cases of perceived length are different from those of Chan's Experiment 3 and our own Experiment 3. Telling participants, in effect, to produce three different reports of perceived length is not the same thing as providing circumstances that bring about three different perceived lengths.

\section{Multicollinearity in Experiments 1-4 of Fitzpatrick et al. (1994)}

The equations for calculating the inertia tensor (see the Appendix) indicate that general manipulations of length, mass, and radius will influence the values of both $I_{1}$ and $I_{3}$. The eigenvalues can, therefore, be highly correlated. This was the case, for example, in Experiments 1-2 of Fitzpatrick et al. (1994), where $I_{1}$ and $I_{3}$ were found to predict perceived length quite reliably. It can be expected to be the case with ordinary objects, as well, whose sizes increase with coincident changes in all dimensions. Do these correlations undermine the statistical import of a reliance on both eigenvalues (cf. Chan, 1995)? There are several reasons that they do not. A high but imperfect correlation does not violate the assumptions of multiple regression; as long as the collinearity is imperfect, estimations of population parameters and tests of statistical significance can proceed (e.g., Berry \& Feldman, 1989). Problems that can arise from imperfect multicollinearity - most specifically, poorly estimated or unstable coefficients (i.e., coefficients that are easily perturbed by a 
Table 4

Degree of Collinearity Between $\log I_{1}$ and $\log I_{3}$ in the Experiments That Show Both to Be Significant, Estimates of Their Exponents (With Confidence Intervals), and Variance Inflation Factors (VIF)

\begin{tabular}{|c|c|c|c|c|c|c|c|}
\hline \multirow[b]{2}{*}{ Experiment } & \multirow[b]{2}{*}{$\begin{array}{c}\text { Number } \\
\text { of Objects }\end{array}$} & \multirow[b]{2}{*}{$\begin{array}{l}\text { Collinearity }\left(r^{2}\right) \text { of } \\
\log I_{1} \text { and } \log I_{3}\end{array}$} & \multicolumn{2}{|c|}{$I_{1}$} & \multicolumn{2}{|r|}{$I_{3}$} & \multirow[b]{2}{*}{$\mathrm{VIF}^{*}$} \\
\hline & & & Exponent & $\begin{array}{c}\text { Confidence } \\
\text { Interval }\end{array}$ & Exponent & $\begin{array}{c}\text { Confidence } \\
\text { Interval }\end{array}$ & \\
\hline FCT E1† & 15 & .87 & .36 & .33 to .40 & -.25 & -.29 to -.22 & 7.69 \\
\hline FCT E2 & 9 & .95 & .37 & .29 to .45 & -.17 & -.30 to -.04 & 20.00 \\
\hline FCT E3 & 9 & .03 & .54 & .51 to .64 & -.34 & -.42 to -.31 & 1.03 \\
\hline FCT E4 & 15 & .28 & .27 & .22 to .31 & -.10 & -.14 to -.05 & 1.39 \\
\hline Our El+ & 9 & .93 & .45 & .29 to .62 & -.30 & -.48 to -.13 & 14.29 \\
\hline S\&T§ & 10 & .80 & .45 & .39 to .51 & -.35 & -.46 to -.23 & 5.00 \\
\hline
\end{tabular}

*VIF < 10 indicates that the estimates of exponents are stable. ${ }^{\dagger}$ FCT, Fitzpatrick, Carello, and Turvey (1994). †Our E1, Experiment 1 of the present series. \$S\&T, Experiments $1-2$ of Solomon and Turvey (1988).

change in the data, such as a deletion of an observation) - can be evaluated. The variance inflation factor (VIF) is one reliable and informative diagnostic. ${ }^{5}$ Table 4 presents an evaluation of the exponent estimates, given the extent of collinearity in the experiments of Fitzpatrick et al. and our own Experiment 1 . Note first the consistent pattern across experiments-a positive exponent for $I_{1}$ and a negative exponent for $I_{3}$ (this was also true within individual subjects). Only in Experiment 2 of Fitzpatrick et al. and our Experiment 1, which had the largest confidence intervals, did VIF exceed 10 , the cutoff for stable coefficient estimates (Montgomery \& Peck, 1990). The particular values of the exponents on the components are not stable, but the significance of the components themselves is reliable.

Even more straightforward is the empirical strategy for combating multicollinearity-specifically, using further data collection in regions of the $x$ space to break up the near collinearities (see, e.g., Berry \& Feldman, 1989; Montgomery \& Peck, 1982, 1990; Ryan, 1990). In Experiment 3 of Fitzpatrick et al. (1994), rods were constructed so as to eliminate the correlation between $I_{1}$ and $I_{3}(r=$ $.17, p>.50$ ). This manipulation was done purposely "to provide a strong test of the generality of the preceding findings [about $I_{1}$ and $I_{3}$ ]" (p. 559). As reported, multiple regression of $\log$ mean perceived length on $\log I_{1}$ and $\log I_{3}$ yielded $r^{2}(8)=.99(p<.0001)$.

\section{The Link Between Actual Length, Perceived Length, and the Tensor: Definite Scaling}

Apart from its profound support for an influence of $I_{3}$, Figure la (Figure 7 from Fitzpatrick et al., 1994) reveals a definite, common scaling (across the four experiments) of length perception to $I_{k}$. Perceivers do not operate in a millimeter or meter range; they have a definite impression of lengths in the tens of centimeters. The tensor sets the scale for how long objects can be perceived to be because it is a meaningful quantification of the objects themselves. Consequently, perceived lengths will order appropriately not only within an experiment but also across experiments, using different observers and different objects. This last point speaks to one of Chan's (1995) motivations for his own experiments--that the fit be- tween actual and perceived length has not been assessed. Relatedly, it speaks to Chan's assertion that previous empirical results do not support the similarity of perceived length to actual length. Almost all of the 50 or so experiments to date on perceived length by dynamic touch have shown that perceived length differs from actual length, more or less. Under some conditions, the match is very close; under others there are large discrepancies (it suffices to look at the results of the nine experiments in Solomon \& Turvey, 1988, and the four experiments of Fitzpatrick et al., 1994). These demonstrations of fits and misfits between perceived and actual length have been integral to the determination of the physical quantity that constrains perceived length in dynamic touch, namely, $I_{i j}$. As noted by Solomon and Turvey (p. 425) in the original series of experiments directed at the nonvisual perception of length by wielding, for any given set of rod lengths, an important restriction on perceived lengths ordering as actual lengths is that the rotational inertias of the rods order in the same way as the lengths of the rods. When this restriction is violated, "shorter rods [should be] perceived as longer than longer rods." This has been a theme of the myriad experiments showing the influence on perceived length of attaching a mass close to or far from the axis of rotation. Further, as noted by Pagano and Turvey (1993, p. 147):

For the objects of the present experiments [rods with cylindrical branches], the structured array of resistances to acceleration in different directions contained information about the locations of the distal tips of the objects to a high degree of metrical accuracy, but it was not perfect. It must be expected for objects constructed in other ways, the specificity of $I_{i j}$ to the maximum linear extents of the objects can be considerably less than that of the present class of objects.

And finally, as suggested by Fitzpatrick et al. (1994, p. 558): "These opposing trends [perception increasing with $I_{1}$ and decreasing with $\left.I_{3}\right]$ keep the perceptions of same-length rods more closely similar over differences in diameter and mass than would be the case if perception scaled only to $I_{1}$ and did so positively."

Although, as Chan (1995) pointed out, the individual experiments of Fitzpatrick et al. (1994) generated some- 
what different power equations, they were strikingly similar in scale (note the exponents on $I_{1}$ and $I_{3}$ provided in Table 4). Despite the fact that they sampled different regions of the $x$ space (e.g., with objects varying in mass from 6 to well over $1,000 \mathrm{~g}$ and lengths anywhere from 22 to $90 \mathrm{~cm}$ ), all data were ultimately accommodated by a single power equation. To those data can be added a reanalysis of Experiments 1 and 2 of Solomon and Turvey (1988), which, in combination, provide a close approximation to the conditions of Chan's Experiment 1: solid aluminum and solid steel rods of fixed diameter and paired lengths. $I_{k}$ for these 10 rods was computed. Due to their wider diameters $(1.25 \mathrm{~cm})$ and greater length range (30-91 cm), even with just two densities, the range of $I_{3}$ was relatively large (the largest was nine times the smallest). Consistent with our reasoning, multiple regression yielded $r^{2}(9)=.99$, with the coefficients on $\log I_{1}$ and on $\log I_{3}$ significant at $p<.0001$ (Partial $F=311$ ) and $p<$ .001 (Partial $F=54$ ), respectively (see the bottom row of Table 4). (Stepwise regression corroborated the need for both eigenvalues.) It is of general theoretical significance to underscore that the data of wide-ranging experiments conform to the regression model $\left(3.8 I_{1}^{41} I_{13}^{-30}\right)$ derived from the data of Fitzpatrick et al.: $r^{2}=.99$ for aforementioned data from Solomon and Turvey (Experiments 1-2), $r^{2}=$ .94 for our Experiment 1, $r^{2}=.96$ for our Experiment 2, and $r^{2}=.90$ for our Experiment 4. This conformity highlights the definite scaling expected from an underlying invariant (Bingham, 1993; Peck, Jeffers, Carello, \& Turvey, 1996; and see below) and counters Chan's (p. 784) suggestion that "knowledge and practice compensate for [the] inadequacy" of the inertia tensor in specifying rod length. Indeed, for what we might refer to as "naturally occurring" objects - that is, those whose structures are not modified (e.g., with handles or attached masses) in order to contrive particular tensors - the power equation for actual length is remarkably similar to the one we see repeatedly for perceived length. For objects that simply vary in diameter, regression of actual length on $I_{1}$ and $I_{3}$ in double logarithmic coordinates yields the power law, actual length $=4.6 I_{1}^{52} I_{3}^{-.51}$. For objects that simply vary in density, actual length $=4.1 I_{1}^{54} I_{3}^{-.54}$. The relationship between actual length and the tensor components underlies the relationship between perceived length and the tensor components (Fitzpatrick et al., 1994).

Recent research has extended the inertia tensor hypothesis (Turvey, Burton, Amazeen, Butwill, \& Carello, in press). When participants made separate judgments about the height and width of a nonvisible rectangular object wielded by means of a handle attached to its base, $I_{1}$ was most significant to perception of length (or, in this case, height), and $I_{3}$ was most significant to perception of width. The same judgments obtained using "tensor objects"3-D cross-like constructions that permit systematic manipulation of $I_{k}$ and, therefore, the inertia tensor, with mass and linear dimensions fixed (Amazeen \& Turvey, 1996) - showed that when $I_{3}$ is varied within a level of $I_{1}$, perceived length decreases, and perceived width increases, with $I_{3}$. Chan (1995) referred to the pattern of decreasing perceived length for fixed $I_{1}$ and increasing diameter in his Experiment 2 as a "diameter-length illusion" that might have some relationship to the size-weight illusion. But we argue that he simply verified the contribution of $I_{3}$ and the scaling provided by its negative exponent. It is noteworthy that weight perception, including the sizeweight illusion, is similarly anchored in $I_{k}$ (Amazeen \& Turvey, 1996). Results of the preceding kind add to those summarized above in demonstrating that the perception of an object's magnitudes by dynamic touch is a function of the object's inertia tensor.

It is very apparent that scaling between perceived and actual length is neither absolute nor relative (Bingham, 1993; Gogel, 1977). An absolute scaling would mean a perfect match every time, which does not occur. A relative scaling would mean that perceived lengths ordered as actual lengths but with the perceived values arbitrarily related to the actual values, which also does not occur. As Bingham noted, when participants can provide judgments that are within a marginal tolerance of the actual values, it must be assumed that there is information available that is more definite than the information supporting relative scaling. He recommended that this type of scaling be called "definite." In perceiving length by dynamic touch, participants exhibit definite scaling-although their perceptions are rarely the actual lengths, they are always in the range of actual lengths (Carello et al., 1992; Turvey \& Carello, 1995; and see summary above). For the 82 objects of Fitzpatrick et al., Solomon and Turvey (1988, Experiments 1-2), and our Experiments 1-2 (i.e., those for which actual length varied), the range of perceived lengths was 23.0 to $94.9 \mathrm{~cm}$, with a mean of $47.9 \mathrm{~cm}$ and a standard deviation of $17.0 \mathrm{~cm}$; the range of actual lengths was 21.2 to $91.4 \mathrm{~cm}$, with a mean of 59.8 and a standard deviation of $21.6 \mathrm{~cm}$. There is a definite scaling of perceived length, which is based, apparently, on the relation between actual length and $I_{k}$.

This last fact is a challenge for the inertia tensor theory of length perception by dynamic touch. Whereas $I_{k}$ is defined uniquely by the mass distribution of a given object, for any given $I_{k}$ there are very many objects, with very different spatial dimensions, that could have given rise to $I_{k}$. Determining an object's length or width from its resistance to rotational acceleration is an "ill-posed" problem, somewhat similar to being given the number 5 and then being asked: "What two numbers were added together to get this number?" (Turvey, 1996). The fact that perceiving length on the basis of $I_{k}$ seems "well posed" from the vantage point of dynamic touch suggests constraints currently hidden from analysis (see Burton \& Turvey, 1990; Carello et al., 1992; Pagano \& Turvey, 1993; Turvey \& Carello, 1995). These constraints are unlikely to be found in a casual appeal to "knowledge and experience." Their identification must be as principled as the rotational dynamics that implicates them. 


\section{REFERENCES}

Amazeen, E. L., \& Turvey, M. T. (1996). Weight perception and the haptic "size-weight illusion" are functions of the inertia tensor. Journal of Experimental Psychology: Human Perception \& Performance, 22, 213-232.

ANDERSON, N. H. (1975). On the role of context effects in psychophysical judgment. Psychological Review, 82, 462-482.

BERRY, W. D., \& FELDMAN, S. (1989). Multiple regression in practice. Newbury Park, CA: Sage.

Bingham, G. (1993). Perceiving the size of trees: Form as information about scale. Journal of Experimental Psychology: Human Perception \& Performance, 19, 1139-1161.

BorisenKo, A. I., \& TARAPOV, I. E. (1979). Vector and tensor analysis with applications (R. A. Silverman, Ed. \& Trans.). New York: Dover.

Burton, G., \& Turvey, M. T. (1990). Perceiving the lengths of rods that are held but not wielded. Ecological Psychology, 2, 295-324.

Carello, C., Fitzpatrick, P., Domaniewicz, I., Chan, T.-C., \& TurVEY, M. T. (1992). Effortful touch with minimal movement. Journal of Experimental Psychology: Human Perception \& Performance, 18, 290-302.

Carello, C., Santana, M.-V., \& Burton, G. (1996). Selective perception by dynamic touch. Perception \& Psychophysics, 58, $1177-1190$.

Chan, T.-C. (1995). The effect of density and diameter on haptic perception of rod length. Perception \& Psychophysics, 57, 778-786.

FitzPatrick, P., Carello, C., \& Turvey, M. T. (1994). Eigenvalues of the inertia tensor and exteroception by the "muscular sense." Neuroscience, 60, 551-568.

GoGel, W. C. (1977). The metric of visual space. In W. Epstein (Ed.), Stability and constancy in visual perception: Mechanisms and processes (pp. 129-181). New York: Wiley.

Goldstein, H. (1980). Classical mechanics. Reading, MA: AddisonWesley.

Hayward, V. (1992). Physical modeling applies to physiology, too. Behavioral \& Brain Sciences, 15, 342-343.

KibbLe, T. W. B. (1985). Classical mechanics. London: Longman.

Montgomery, D. C., \& PECK, E. A. (1982). Introduction to linear regression analysis. New York: Wiley.

Montgomery, D. C., \& Peck, E. A. (1990). Multicollinearity in regression. In H. M. Wadsworth (Ed.), Handbook of statistical methods for engineers and scientists (pp. 15.1-15.23). New York: McGraw-Hill.

MOON, P., \& SPENCER, D. (1986). Theory of holors: A generalization of tensors. Cambridge: Cambridge University Press.

Pagano, C. C., Carello, C., \& Turvey, M. T. (1996). Exteroception and exproprioception by dynamic touch are different functions of the inertia tensor. Perception \& Psychophysics, 58, 1191-1202.

Pagano, C. C., Fitzpatrick, P., \& Turvey, M. T. (1993). Tensorial basis to the constancy of perceived object extent over variations of dynamic touch. Perception \& Psychophysics, 54, 43-54.

Pagano, C. C., Kinsella-Shaw, J., Cassidy, P., \& Turvey, M. T. (1994). Role of the inertia tensor in haptically perceiving where an object is grasped. Journal of Experimental Psychology: Human Perception \& Performance, 20, 276-285.

Pagano, C. C., \& Turvey, M. T. (1992). Eigenvectors of the inertia tensor and perceiving the orientation of a hand-held object by dynamic touch. Perception \& Psychophysics, 52, 617-624.

Pagano, C. C., \& Turvey, M. T. (1993). Perceiving by dynamic touch the distances reachable with irregular objects. Ecological Psychology, 5, 125-151.

Peck, A., Jeffers, L., Carello, C., \& Turvey, M. T. (1996). Perceiving the length of one rod by means of another. Ecological Psychology, $8,237-258$.

RyaN, T. P. (1990). Linear regression. In H. M. Wadsworth (Ed.), Handbook of statistical methods for engineers and scientists (pp. 13.113.37). New York: McGraw-Hill.

Sinedecor, G. W., \& Cochran, W. G. (1989). Statistical methods. Ames: Iowa State University Press.

Solomon, H. Y., \& TURVEY, M. T. (1988). Haptically perceiving the distances reachable with hand-held objects. Journal of Experimental Psychology: Human Perception \& Performance, 14, 404-427.
Solomon, H. Y., Turvey, M. T., \& Burton, G. (1989a). Gravitational and muscular variables in perceiving extent by wielding. Ecological Psychology, 1, 256-300.

Solomon, H. Y., Turvey, M. T., \& Burton, G. (1989b). Perceiving extents of rods by wielding: Haptic diagonalization and decomposition of the inertia tensor. Journal of Experimental Psychology: Human Perception \& Performance, 15, 58-68.

SPENCER, D. (1991). Vector and tensor analysis. In R. G. Lerner \& G. L. Trigg (Eds.), Encyclopedia of physics (pp. 1334-1337). New York: $\mathrm{VCH}$.

SPROTT, J. C. (1991). Numerical recipes: Routines and examples in basic. New York: Cambridge University Press.

Stevens, S. S. (1971). Issues in psychophysical measurement. Psychological Review, 78, 426-450.

TANGHERLINI, F. R. (1991). Moment of inertia. In R. G. Lerner \& G. L. Trigg (Eds.), Encyclopedia of physics (pp. 767-770). New York: VCH.

TURveY, M. T. (1996). Dynamic touch. American Psychologist, 51, $1134-1152$.

Turvey, M. T., Burton, G., Amazeen, E., Butwill, M., \& Carello, C. (in press). Perceiving the width and height of hand-held objects by dynamic touch. Journal of Experimental Psychology: Human Perception \& Performance.

Turvey, M. T., Burton, G., Pagano, C., Solomon, H. Y., \& RuneSON, S. (1992). Role of the inertia tensor in perceiving object orientation by dynamic touch. Journal of Experimental Psychology: Human Perception \& Performance, 18, 714-727.

Turvey, M. T., \& Carello, C. (1995). Dynamic touch. In W. Epstein \& S. Rogers (Eds.), Handbook of perception and cognition: Vol. 5. Perception and motion (pp. 401-490). New York: Academic Press. WEDELL, D. H. (1995). Contrast effects in paired comparisons: Evidence for both stimulus-based and response-based processes. Journal of Experimental Psychology: Human Perception \& Performance, 21, 1158-1173.

\section{NOTES}

1. We use the symbol $I_{i j}$ for the inertia tensor rather than the symbol I used by Chan (1995) and Fitzpatrick et al. (1994). $I_{i j}$ is preferred by most authors because the index notation is the basis for tensor transformations, tensor algebra, and tensor equations (e.g., Borisenko \& Tarapov, 1979; Moon \& Spencer, 1986). The entries by Tangherlini (1991) and by Spencer (1991) in the Encyclopedia of Physics summarize, respectively, the modern perspectives on the inertia tensor and tensors in general.

2 . The same results are obtained with a variety of techniques for redefining regressors. Backward elimination, stepwise regression, and the method of all possible regressions (using Mallows's $C_{p}$ statistic; see Ryan, 1990; Snedecor \& Cochran, 1989) were used to reanalyze each experiment separately and data from all four together. Diameter (in the form of $\log d$ ) was considered along with $I_{1}$ and $I_{3}$. A single technique preferred $\log d$ over $\log I_{3}$ in a single experiment. Especially telling is a comparison of Experiment 1, in which diameter was varied and density was fixed, and Experiment 2, in which diameter was fixed and density was varied. According to the diameter hypothesis, the outcomes of these two experiments should have been very different. Quite to the contrary, the data from the two experiments were indistinguishable.

3. The values of $I_{3}$ were recalculated rather than being taken from Chan's (1995) Table 1. Chan referred to the maximum and minimum principal moments, that is, the major and minor eigenvalues $I_{1}$ and $I_{3}$, but in fact used only the coordinate-system dependent moments of inertia $I_{x x}$ and $I_{y y}$ (see his Figure 1). This oversight stems from a misconception expressed on p. 778 that $I_{x x}$ and $I_{y y}$ are the principal moments for rotations centered at the wrist as long as the rotations are restricted to the $y z$ plane-that is, about a single axis (see his Figure 1). Even if his participants had been so restricted (which they were apparently not; see Chan, 1995, p. 780), in actual fact, there will always be products of inertia whenever the fixed rotation point does not lie on a principal central axis of inertia, defined about the center of mass (see Appendix). The relevant principal moments or eigenvalues will have to be determined by diagonalizing the inertia tensor matrix (Fitzpatrick et al., 
1994; Pagano et al., 1993; Pagano, Kinsella-Shaw, Cassidy, \& Turvey, 1994; Turvey et al., 1992). For the situation depicted in Chan's Figure 1, the geometric interpretation of diagonalization is a rotation of the coordinate system counterclockwise, bringing the $y$-axis closer to the rod and the (negative) $z$-axis further from the rod. This rotation results in a decrease in the smallest of the three moments, that defined about $y$, and an increase in the moment of intermediate value, that defined about $z$. The result is $I_{3}<I_{y y}$ and $I_{2}>I_{z z}$, with $I_{1}=I_{x x}$ because there is no change in the orientation of the $x$ axis.

4. Interestingly, a multiple regression of $\log$ perceived length on $\log I_{1}$ and $\log$ diameter revealed both components to be significant $(p<.0001$ and $p<.05$, respectively). Patently, this cannot be a contribution of grasp diameter, which is constant due to the use of handles. We suspect that it is standing proxy for the tensorial contributions, in effect amplifying the range of $I_{3}$ values.

5. The VIF is given by $C_{i j}$, defined as

$$
C_{j j}=\left(1-R_{j}^{2}\right)^{-1}
$$

where $R_{j}^{2}$ is the coefficient of determination resulting from regressing $x_{j}$ on the remaining $p-1$ regressors (Montgomery \& Peck, 1990). If $x_{j}$ is nearly orthogonal to the remaining regressors, then $R_{j}^{2}$ is small and $C_{j j}$ is close to unity; if $x_{j}$ is nearly nonorthogonal, then $R_{j}^{2}$ is near unity and $C_{j j}$ is large. $\mathrm{A} C_{j j}$ in excess of 10 means that the coefficients are poorly estimated (Montgomery \& Peck, 1990). Specifically, $C_{i j}$ is the amount by which the variance of the regression coefficient associated with $x_{j}$ is inflated due to near collinearity among the regressors; a direct measure of the increase in the coefficient's confidence interval due to near multicollinearity is provided by $C_{j j}^{1 / 2}$.

\section{APPENDIX}

When an object is held firmly in the hand and wielded about a fixed point (by means of movement at the wrist, elbow, shoulder, or combinations of such motions), motions on the part of the object and torques on the part of the muscles vary over time. The object can be moved quickly or slowly, with large or small amplitudes; the movement can be forceful or easy. These motions and torques are linked, however, by a time-invariant quantity, the inertia tensor, $I_{i j}$ (Fitzpatrick et al., 1994; Pagano et al., 1994; Solomon \& Turvey, 1988; Turvey \& Carello, 1995). $I_{i j}$ is a $3 \times 3$ symmetric matrix (Figure Ala). Because it is symmetric - the three numbers below the diagonal equal the three numbers above the diagonal-an object's mass distribution can be captured in six components. Three of these are moments of in- ertia $\left(I_{x x}, I_{y y}\right.$, and $\left.I_{z z}\right)$ on the diagonal and three are products of inertia $\left(I_{x y}, I_{y z}\right.$, and $\left.I_{x z}\right)$ off the diagonal. Calculations are with respect to a rectangular coordinate system $O x y z$ anchored in the wrist (Figure Alb), so that the moments are the resistances to rotational acceleration about the three orthogonal axes (i.e., the moment of the inertia forces opposing the torque's tangential components), and the products are the resistances in directions perpendicular to these rotations (i.e., the moment of the inertia forces opposing the torque's radial components). Equations for homogeneous solid cylinders illustrate the consequences of experimental manipulations such as object length, mass, radius, and grip location:

$$
\begin{aligned}
& I_{x x}=\left(m r^{2 / 4}+m L^{2 / 12}\right)+m\left(y^{2}+z^{2}\right), \\
& I_{y y}=\left(m r^{2} / 4+m L^{2 / 12}\right)+m\left(x^{2}+z^{2}\right), \\
& I_{z z}=\left(m r^{2} / 2\right)+m\left(x^{2}+y^{2}\right), \\
& I_{x y}=-m x y, \\
& I_{y z}=-m y z,
\end{aligned}
$$

and

$$
I_{x z}=-m x z
$$

where $m$ is the rod mass, $r$ is the rod radius, $L$ is the rod length, and $x, y$, and $z$ are perpendicular distances along the axes to Oxyz. The first term in each moment equation is $I_{i j}$ through the center of mass (CM); the second term, in accordance with the parallel axis theorem, reflects the additional resistance when $O$ is not at the CM. The products reflect the asymmetry of the mass distribution with respect to $O$. For a cylindrical rod, asymmetry is a consequence of grip location (Carello, Santana, \& Burton, 1996; Pagano, Carello, \& Turvey, 1996; Pagano et al., 1994). For rods grasped in the hand, as in Figure A1b, $x=0, y$ is approximately $6 \mathrm{~cm}$ (the perpendicular distance from $O$ in the wrist to the longitudinal axis of the rod), and $z$ indicates how far $O$ is forward or back of the rod CM. Unless $O$ and $C M$ coincide, for which both $x$ and $z=0, I_{y z}$ is nonzero.

For convenience, we have chosen to align the $z$-axis with the longitudinal axis of the rod, with $x$ running horizontally and $y$ vertically. But this choice is not required mathematically; an infinite number of 3-axis sets can be anchored at $O$, and the components of $I_{i j}$ will differ for each. There is an invariant set, how-
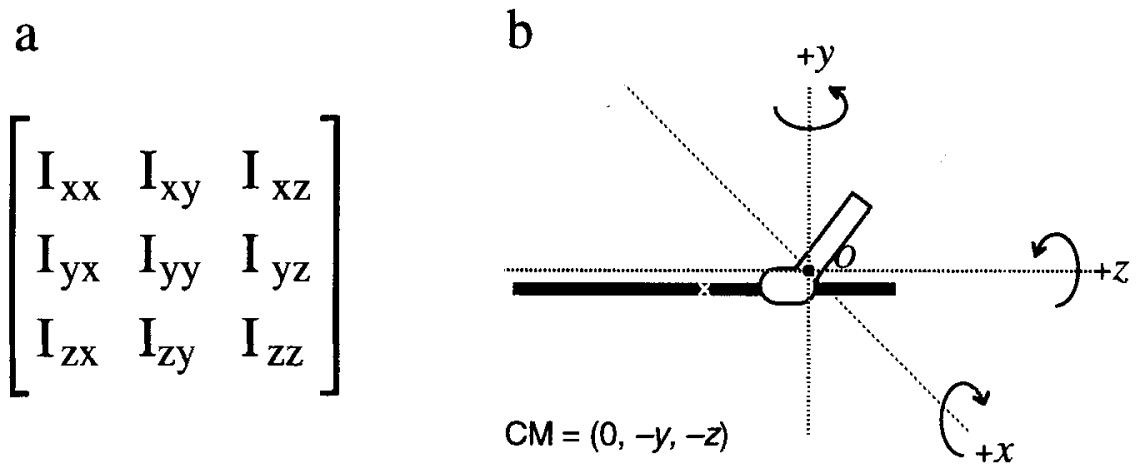

Figure A1. (a) The inertia tensor $I_{i j}$ is a hypernumber that quantifies the mass distribution of an object. (b) A wielded rod resists rotation about the $x$-, $y$-, and $z$-axes, which define a coordinate system centered at a point $O$ in the wrist. The coordinates of the rod's center of mass (CM) indicate which products are nonzero-in this case, only that product involving the $y$ and $z$ coordinates. 
a

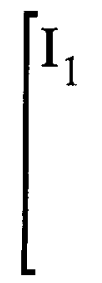

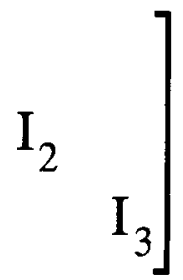

b

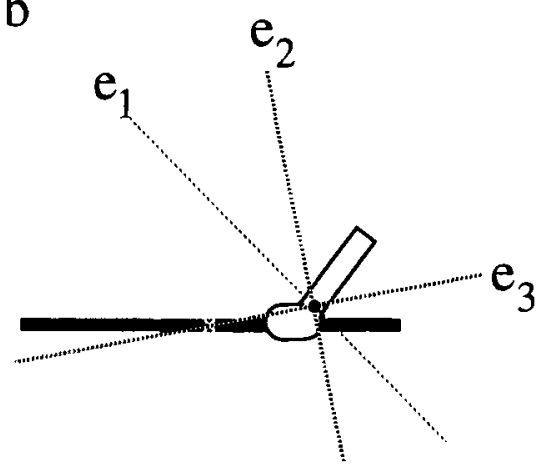

Figure A2. The diagonalized form is with respect to the symmetry axes of the object about the point of rotation. (a) The eigenvalues $I_{k}$ are the principal moments that remain when the products of inertia have been eliminated. (b) The eigenvectors $e_{k}$ are the directions of the new axes.

ever, that is independent of $O x y z$. The so-called diagonalized form is obtained by orienting the axes so as to eliminate the products of inertia (Figure A2). These new orientations are the principal axes or eigenvectors of the tensor. The lengths of the eigenvectors are related to the principal moments or eigenvalues of the tensor (as $1 / \sqrt{I_{i j}}$ ). Although $I_{i j}$ from all of the other coordinate systems are related to the diagonalized form by a transformation law (see, e.g., Borisenko \& Tarapov, 1979), the great advantage of eigenvalues and eigenvectors is that they are independent of the coordinate system (Hayward, 1992; Turvey \& Carello, 1995).

(Manuscript received October 2, 1995; revision accepted for publication November 14, 1996.) 